\title{
Real Time Vehicle Tracking Algorithm Based on Statistical Characteristics in HSI Color Model
}

\author{
Pramod Sethy $^{1 *}$, Gaurav Singh ${ }^{2}$ and Karuna Lochab ${ }^{3}$ \\ Krishna Engineering College Ghaziabad, India \\ pramodtuku@gmail.com,gaurav.singh@krishnacollege.ac.in \\ karuna.lochab@krishnacollege.ac.in
}

\begin{abstract}
Image processing and capturing has become a crucial part of intelligent robotics. It is mainly used to detect an object in real-time. To control traffic on the roads, there is a need for well-informed traffic management system. License plate spying can be used in wide domains such as for detecting speeding vehicles, traffic rule enforcement, security control and collect toll electronically. License plate detection can be performed via various approaches such as vector quantization, Gabor transform, optical character recognition, neural networks etc. Character recognition in license plate is an inevitable factor of Real Time vehicle Tracking (RTVT) system. Lot of research has been carried out to find the characters in the vehicle license plate. In [2], the character recognition is done by extracting the plate region of the license plate by using edge detection algorithms and smearing algorithms. Then image segmentation is done by using smearing algorithms, filtering techniques and some morphological algorithms are also used. Lastly, statistical approach is used to identify the characters in the license plate using template matching. This project will focus on implementing an algorithm in [4], which uses hue, saturation, intensity (HSI) color model to determine the threshold statistically for detecting the candidate regions. Irrespective of the color of the license plate, the candidate regions shall include license plate regions and the geometrical properties are used for classification of the license plates. Predetermined license plate alphanumeric characters shall be used to decompose the candidate regions by using 2-D cross correlation and detect the license plate. The effectiveness of the algorithm will be studied followed by conclusions.
\end{abstract}

Keywords: HSIcolor model, Gabor transform, Optical Character recognition, neural networks

\section{Introduction}

The aim of making out a specific object in an image is challenging in digital image processing. With the rapid development in the automobile industry and increasing traffic, intelligent transport systems gained significant interest. Intelligent transport system refers to combining information and communication technology to transport infrastructure with the aim of improving traffic, vehicle wear and tear and fuel consumption. The technology based systems in intelligent transport system are basically divided into intelligent infrastructure systems and intelligent vehicle systems [5]. Computer vision and character recognition algorithms for Real Time Vehicle Tracking (RTVT) are vital modules for intelligent infrastructure systems. Real Time Vehicle Tracking (RTVT) is an active research area under intelligent transport system. RTVT should operate online in a real-

* Corresponding Author 
time basis so as satisfy the needs of intelligent transport system. RTVT is a challenging task because different vehicle images are taken in different sets of conditions like nonuniform outdoor illumination during data acquisition, different forms of vehicle license plate formats and orientation of the vehicle license plate in the image due to difference in the angle subtended between the camera and object of interest. RTVT is widely used to detect speeding cars, security control in restricted areas and for traffic law enforcement. Detecting the license plate region and performing character recognition is an important research topic of RTVT system.

Generally, RTVT algorithms are composed of three processing steps that include locating the license plate in the given image, segmentation of the plate characters and recognition of each character. The first two steps are implemented using image processing on still images or videos. Due to exponential increase in the technology, recent developments indicate that it takes less than $50 \mathrm{~ms}$ for plate detection and recognition with the ability of processing more than 20 frames per second for videos [5]. Although there are numerous techniques reported in literature for RTVT, there is still a need for a common platform where different methods can be evaluated based on performance, execution time, percentage of false alarm, percentage of detection and recognition rate. Hence it is inappropriate to declare which methods give the highest performance. The focus of this project is to determine the threshold statistically using HIS color model to binarize the image and then perform character recognition in MATLAB. This method detects license plate having a white background with red colored characters engrossed on the plate, when the angle subtended between the camera and the object of interest is almost perpendicular. This means that the license plate should not be tilted. Furthermore, the candidate regions are eliminated using area of the license plate and template matching is done to the characters extracted (recognized) from the license plate to identify the characters.

\section{Real Time Vehicle Tracking Modules}

The RTVT consist of three main modules. The first part deals with the conversion of the RGB colored image to HSI image, detection of the candidate region i.e. the license plate. The second part defines procedures for refining the candidate regions using edge detection algorithms, labeling and filtering. Geometrical properties such as area of the license plate can be used to filter the candidate like regions. The last part is the character extraction of the license plate and template matching to identify the characters in the vehicle license plate.

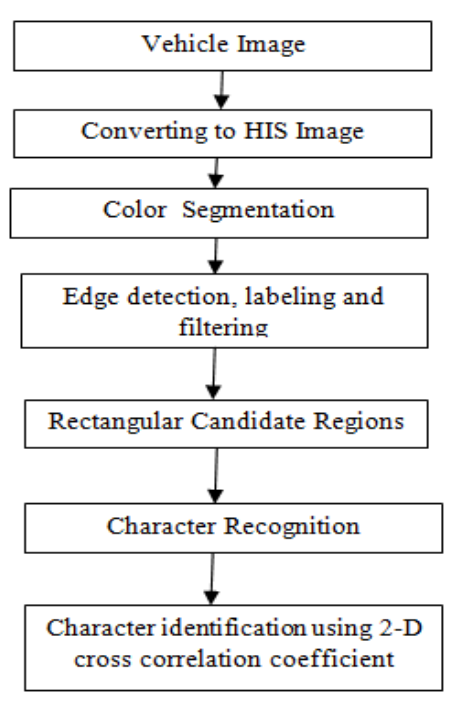




\subsection{Color Segmentation}

Input vehicle images that are in RGB color module is converted to HSI color module. The candidate regions are found using the saturation, hue and intensity values of the acquired RGB image.

\subsection{RGB Color Model}

RGB model is comprised of three basic colors namely red, green and blue. Resultant color is generated by superimposing the spectral components of RGB colors. In general, it describes color as seen from human perspective. The receptors present in the retina of the human eye are highly sensitive to the primary colors of RGB model. Primary colors of RGB model are represented within a cubic volume by orthogonal axes of red, green and blue color respectively, as shown in Figure 6.2. Origin of this coordinate system denotes black color where coordinate system corresponds to value $0(\mathrm{R}=\mathrm{G}=\mathrm{B}=0)$, while white color is present at the opposite corner of cube having coordinate values equal to $255(\mathrm{R}=\mathrm{G}=\mathrm{B}=255)$. The line connecting black and white color points is the diagonal.

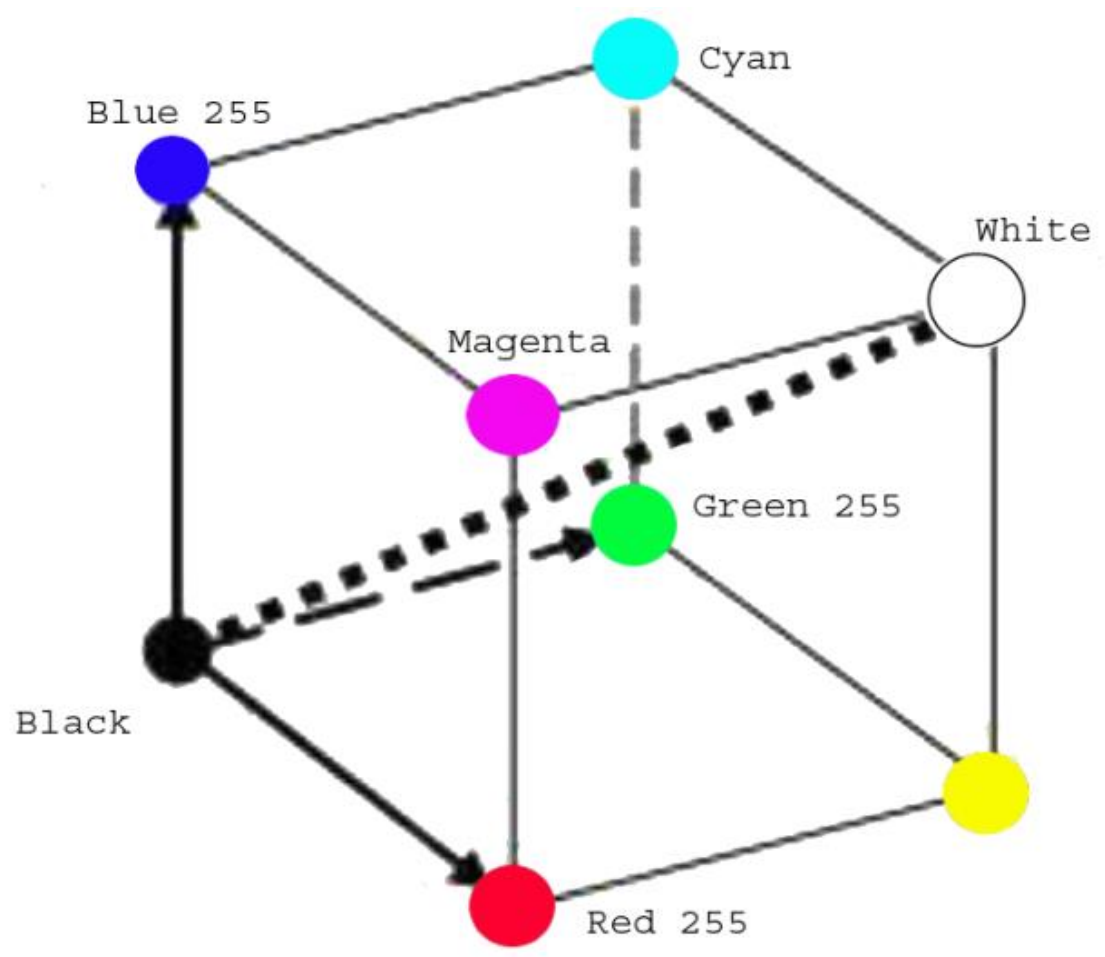

Figure 2.2. RGB Color Model [2]

\subsection{HSI Color Model}

Basically HSI colors are not described by the percentage composition of the primary colors but rather by their saturation, hue and intensity. The saturation is defined as the pureness of the color, the hue represents the color itself and the intensity represents the brightness of the color. All color components can be separated using the saturation, hue and intensity values. As seen in the Figure 6.3, the HSI model presents colors within a double-cone space. The hue is defined as angle 0 , ranging from $0^{\circ}$ to $360^{\circ}$. Adjusting the hue, colors will vary from red color at $0^{\circ}$, through yellow color at $60^{\circ}$, green color at $120^{\circ}$, blue color at $240^{\circ}$ and again back to red at $360^{\circ}$. Saturation ' $\mathrm{S}$ ' is represented through the radius that could range from 0 to 1 . When $S$ value is 0 , the resultant color is 
gray of intensity 1 . When $\mathrm{S}$ value is 1 , the resultant color is on the top of the conical base. On any horizontal slice through the model space, the hue (or "color" of the color) varies through the slice, and the saturation value (the purity of the color) increases radially outwards from the central intensity axis. The vertical axis represents intensity, which predicts variations in the darkness and lightness of any color. The zero intensity level defines black color whereas the full intensity level defines white color. HSI values elsewhere along the intensity axis represents different levels of gray color. Intensity makes no contribution to the color, in the HSI color model.

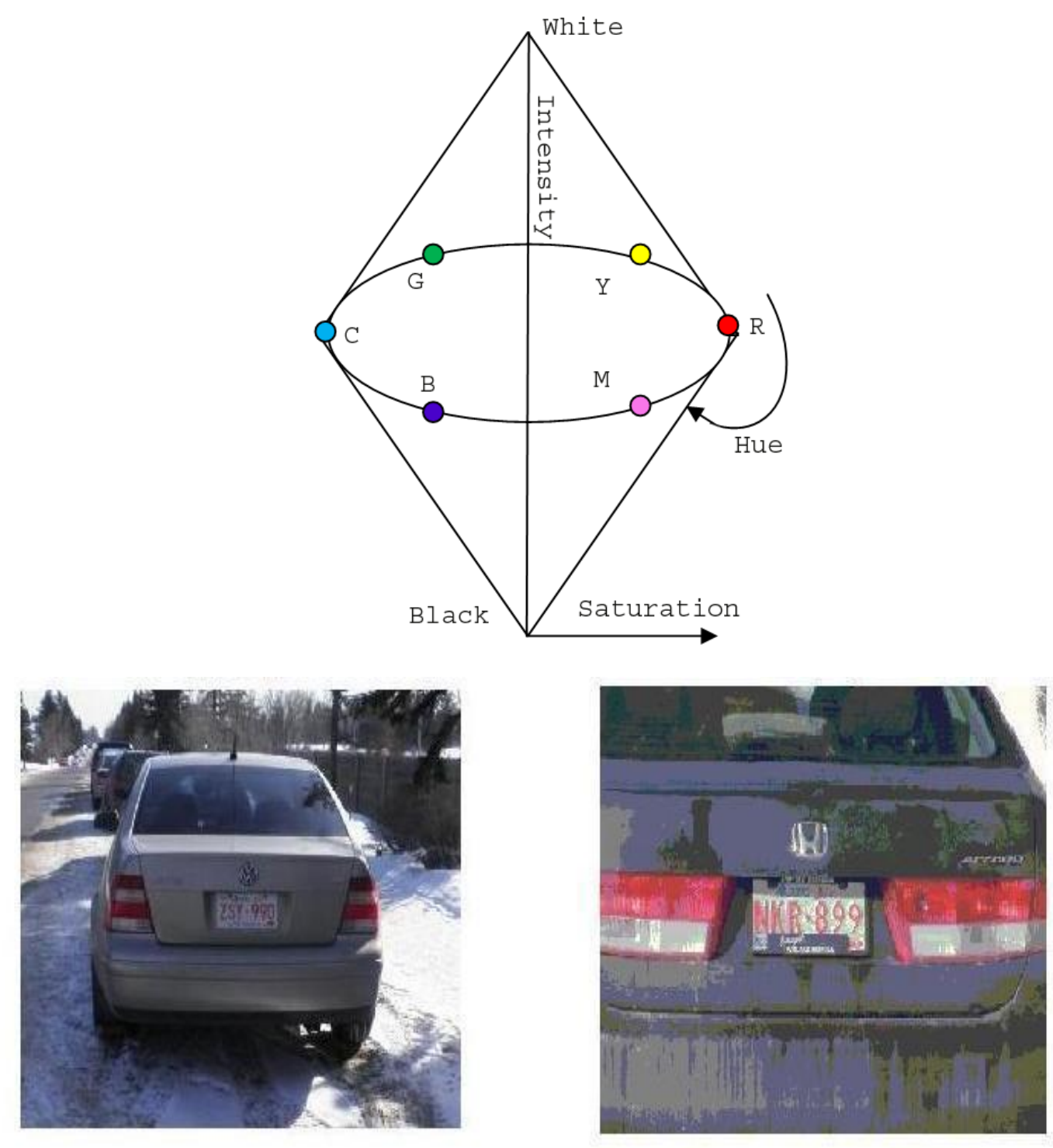

Figure 2.4 RGB Car Image

The following transformations equations are employed to transform an RGB image to HSI image are as below. 
$H=\cos ^{-1}\left\{\frac{0.5[(R-G)]+(R-B)}{\left[(R-G)^{2}+[(R-B)(G-B)]\right]^{\frac{1}{2}}}\right\}$

$S=1-\frac{3}{(R+G+B)} \min (R, G, B)$

$I=\frac{R+G+B}{3}$

Figure 2.5, shows HSI car image.
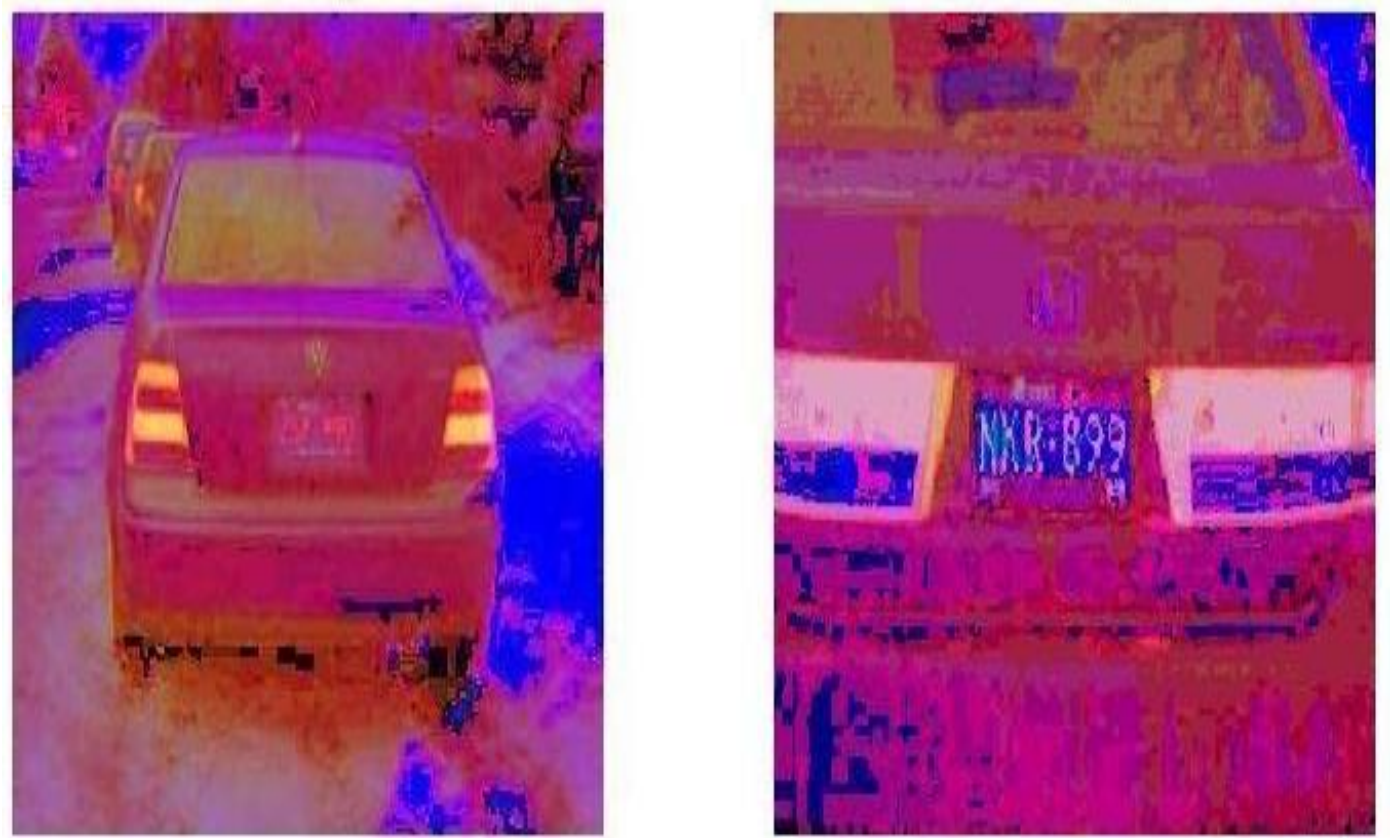

Figure 2.5. HSI Car Image

In the implementation of this project, the license plate detection is based on the color properties of the plate i.e., mean value and standard deviation value of the hue. To detect white pixels in the license plate, hue has got no meaning. Binarization results in an image whose pixels have only two possible values, 0 (black) and 1 (white) Figure 2.6. The thresholding method used here is the Otsu algorithm [14-15], which is based on the assumption that the image to be thresholded comprises of two classes of pixels, it then calculates the value of optimum threshold separating the two classes such that the combined spread is minimal. Hence saturation along with intensity parameters are taken into account. To determine mean and standard deviation, 35 sample images were taken. Mean and standard deviation values for saturation and intensity is computed for white pixels in the sample set of images whereas mean and standard deviation of hue is computed to detect red pixels in the license plate from the same sample of images. For detecting the red pixels, the binarization procedure can be formulated as below,

$b_{\text {RED }}=\left\{\begin{array}{l}1,\{[\mu H-\sigma H \leq H(x, y) \leq \mu H+\sigma H] \&[S(x, y) \geq 0.03] \&[0.05 \leq I(x, y) \leq 0.95]\} \\ 0, \text { otherwise }\end{array}\right\}$ 
Where $\mathrm{S}(\mathrm{x}, \mathrm{y}), \mathrm{H}(\mathrm{x}, \mathrm{y})$ and $\mathrm{I}(\mathrm{x}, \mathrm{y})$ are saturation, hue and intensity components of the $\mathrm{x}^{\text {th }}$, $\mathrm{y}^{\text {th }}$ pixel respectively. $\sigma \mathrm{H}$ and $\mu \mathrm{H}$ are the standard deviation and mean of hue calculated from the data sample respectively. The binarization procedure for detecting the white pixels in license plate is formulated below where $\mathrm{I}(\mathrm{x}, \mathrm{y}), \mathrm{S}(\mathrm{x}, \mathrm{y})$ are intensity and saturation components of $x^{\text {th }}, y^{\text {th }}$ pixel respectively. $\mu \mathrm{S}, \sigma \mathrm{S}$ presents the mean and standard deviation of saturation values of the data sample respectively. Whereas $\sigma \mathrm{I}, \mu \mathrm{I}$, are the standard deviation and mean of the intensity values of the data sample respectively. Figure 2.6 shows binary image after color segmentation Color segmentation can result in detection of all possible candidate regions from the input image. False candidates will be filtered out in the labeling stage and filtering stage as

$$
b_{\text {WHITE }}=\left\{\begin{array}{l}
1,\{[S(x, y) \leq \mu S+\sigma S] \&[I(x, y) \geq(\mu I+0.25+\sigma I)]\} \\
0, \text { otherwise }
\end{array}\right\}
$$

\subsection{Morphological Processing}

After segmentation, there may be some noise in the image such as small holes in the candidate regions. This could be resolved with morphological processing. Mathematical morphology operations are based on the shape in the image and not pixel intensities. The two basic morphological operations available in MATLAB are dilation and erosion.
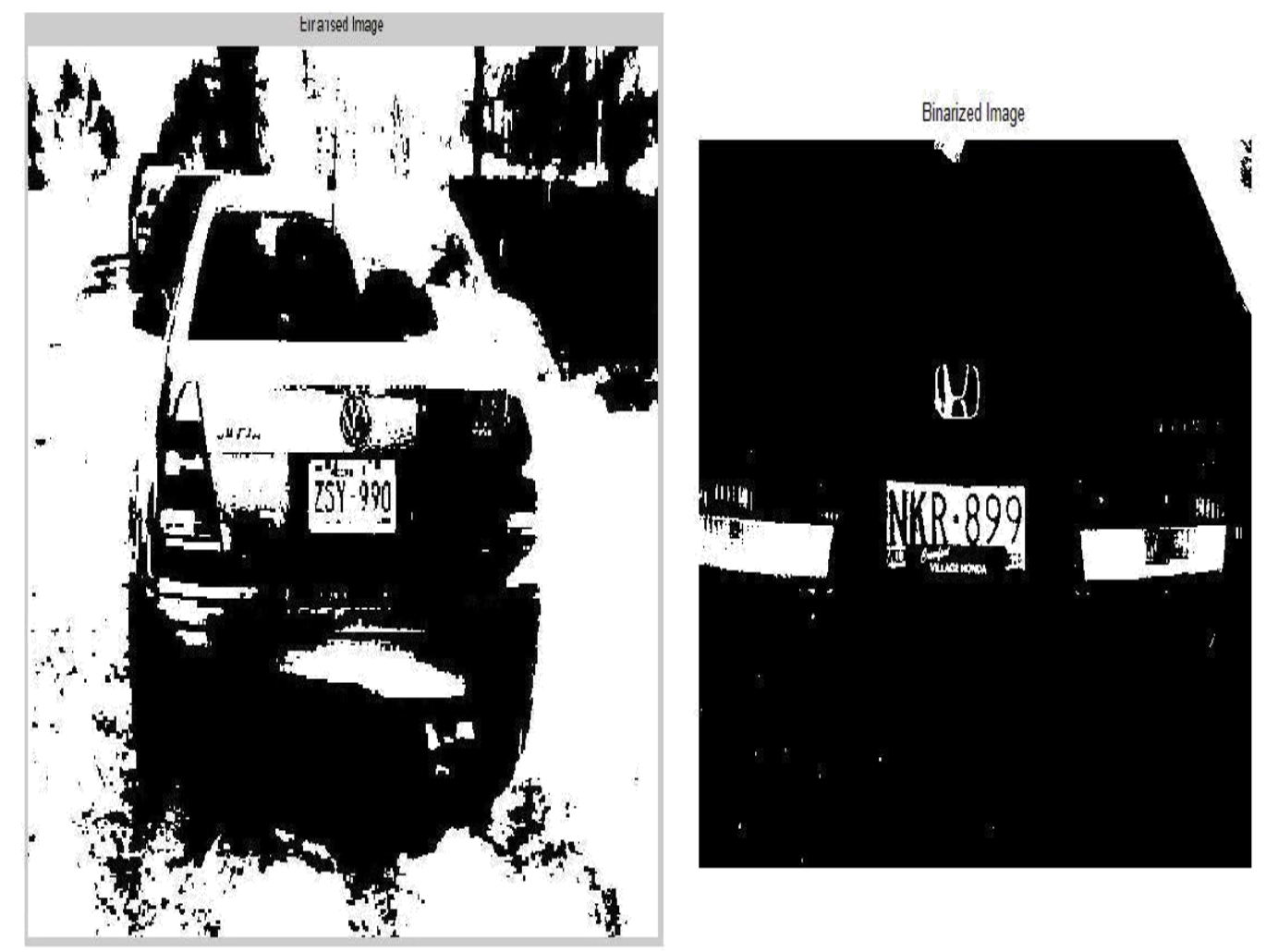

Figure 2.6. Binary Image after Color Segmentation

Dilation causes objects to expand while erosion allows objects to shrink by eroding the object boundaries. These components can be modified by careful choice of the structuring element to determine objects which will be dilated or eroded. Structuring element is defined by a matrix of 0 s and $1 \mathrm{~s}$ which could be of any arbitrary size and shape. In 
MATLAB one can define neighborhood of desired size for the structuring element such as square, rectangle, diamond etc. Preferably rectangle is used as the neighborhood for the structuring element of size $6 \times 4$ which is obtained by a trial and error method. In the project, closing operation is used which is dilation followed by erosion. Removal of small holes present in the plate plays an important role in obtaining the rectangular license plate. Figure 2.7, shows morphological operation on binary image.
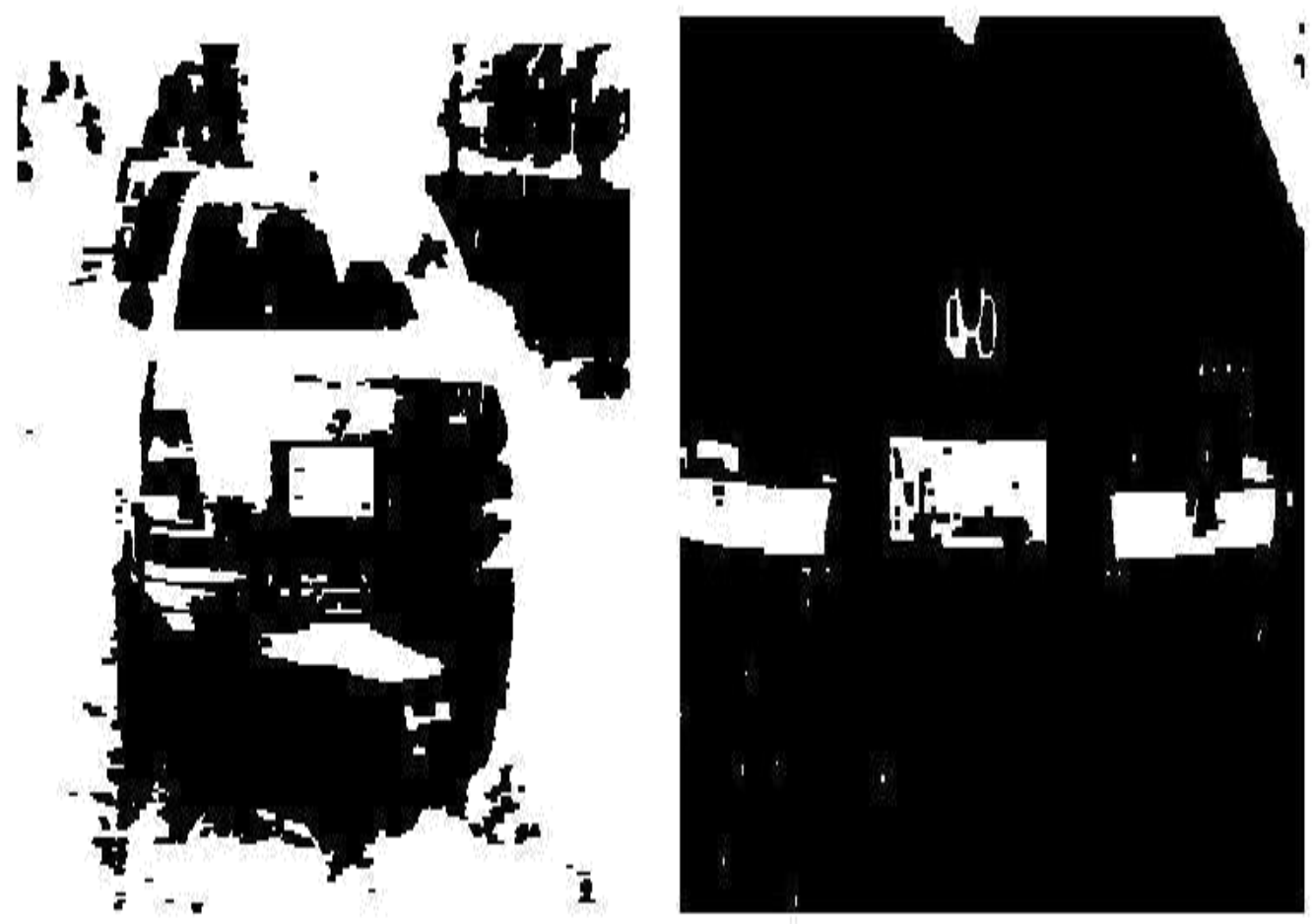

Figure 2.7. Morphological Processing

\subsection{Edge Detection, Labeling and Filtering}

Edge detection is one of the major task for digital image processing. Edge points possess high gradient difference in the local neighborhood. They are used for feature extraction in image processing. Considerably the image date is reduced after edge detection. Some popular edge detectors [10] are Sobel, Robert's Cross, Prewitt, Kirsch, Laplace and Marr-Hildreth. Edges are described as intensity gradients within the image. In this paper, Sobel edge detection operator is implemented in MATLAB. Sobel operator is basically a combination of two operators: one detects horizontal edges and the other detects the vertical edges. It is a $3 \times 3$ neighborhood based gradient operator. The resultant image obtained after applying Sobel operator is shown below in Figure 2.8. 

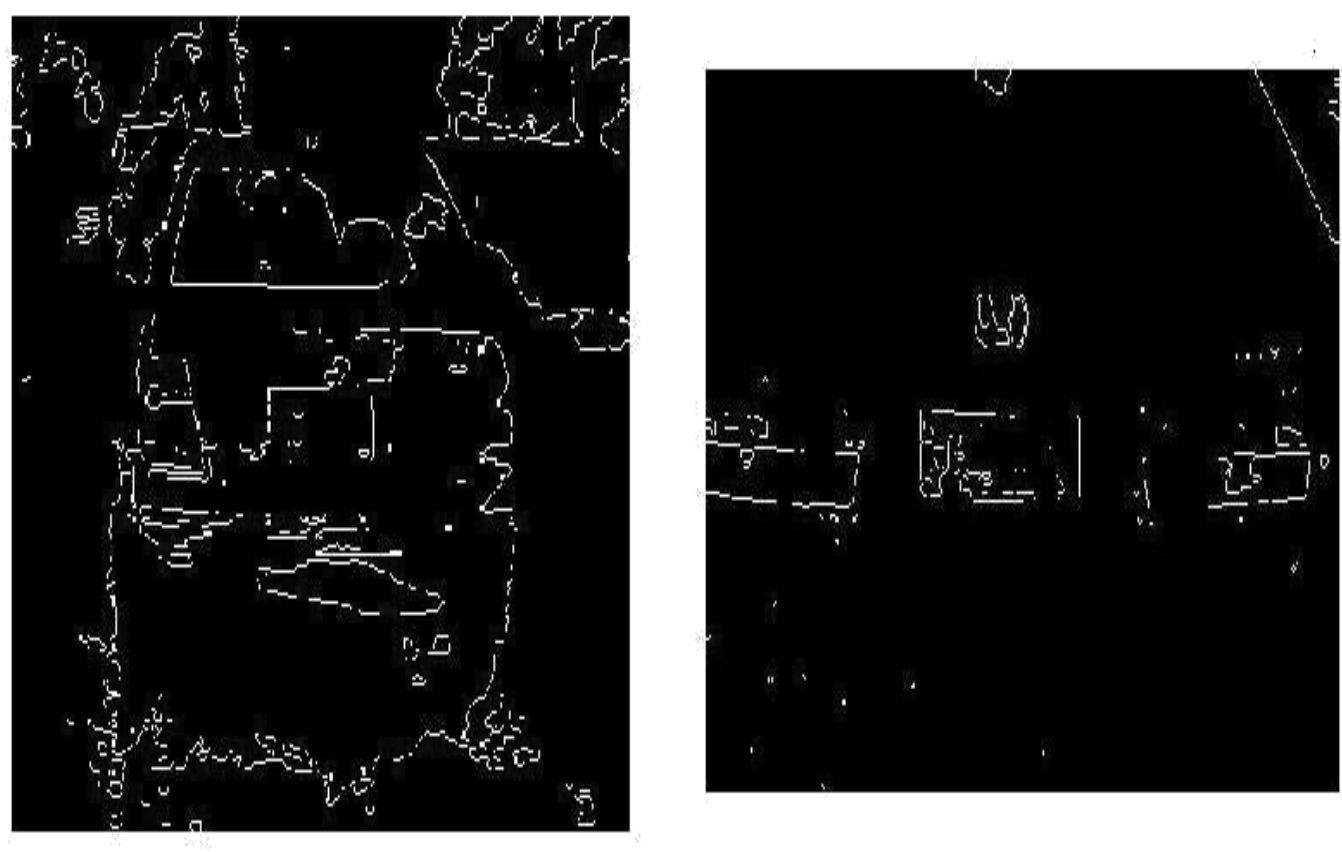

Figure 2.8. Edge Detection- Sobel Operator

After detecting the candidate regions using color segmentation and edge detection, features of the region have to be extracted to differentiate the LP regions from other regions. So the next step in the algorithm is to label each of the connected components. Before that the image is smoothened so as to reduce the number of connected components. To smooth an image is to create an approximate image that attempts to capture the important patterns in the data, while leaving out noise and other fine-scale structures/rapid phenomena. The smoothened image is as shown in Figure 2.9.
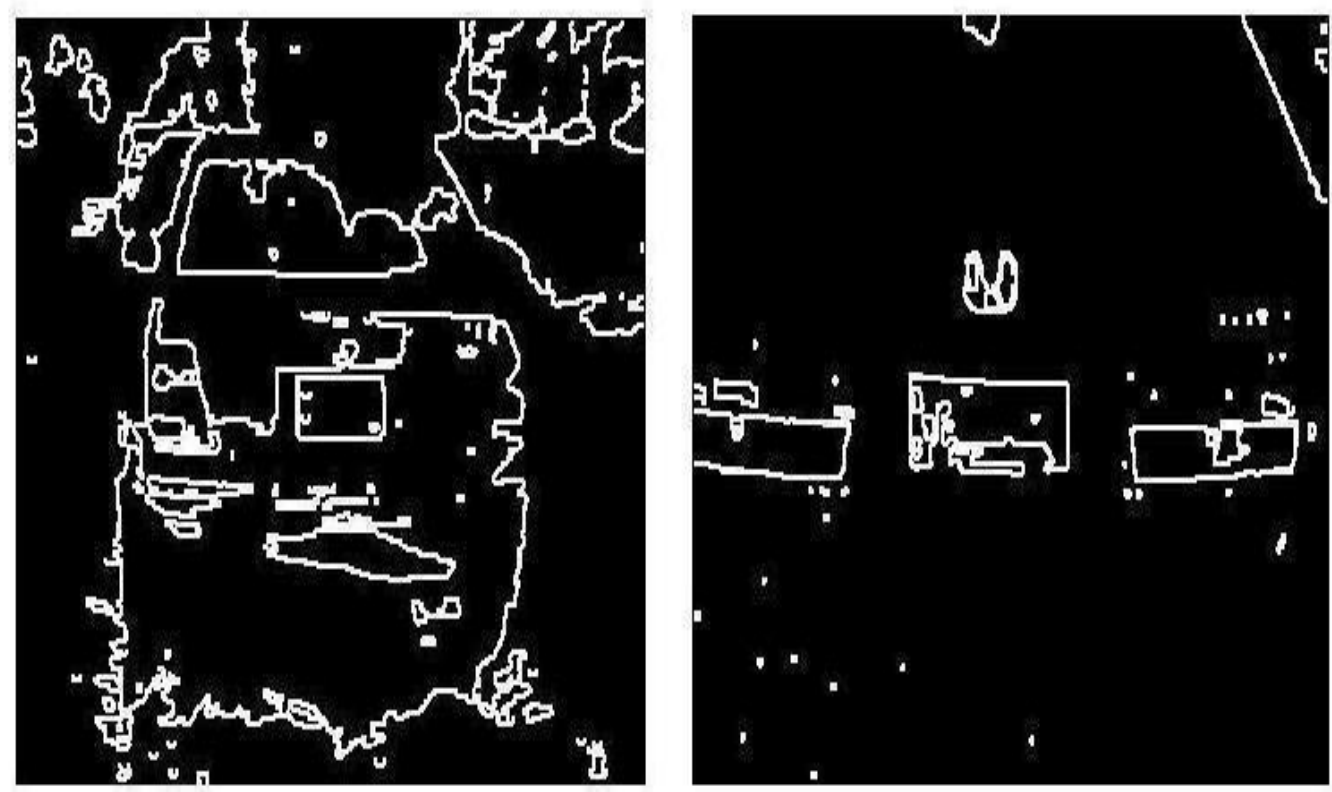

Figure 2.9. Smoothened Image 
The connected components are labeled. It works on one connected component at a time and can move all over the image. During this step, area of the connected component is used to filter candidate like regions for detecting the license plate. A trial and error method was used to study each connected component namely its area, aspect ratio. This could be used to filter candidate like regions in the license plate so as to obtain the rectangular region as shown in Figure 2.10.
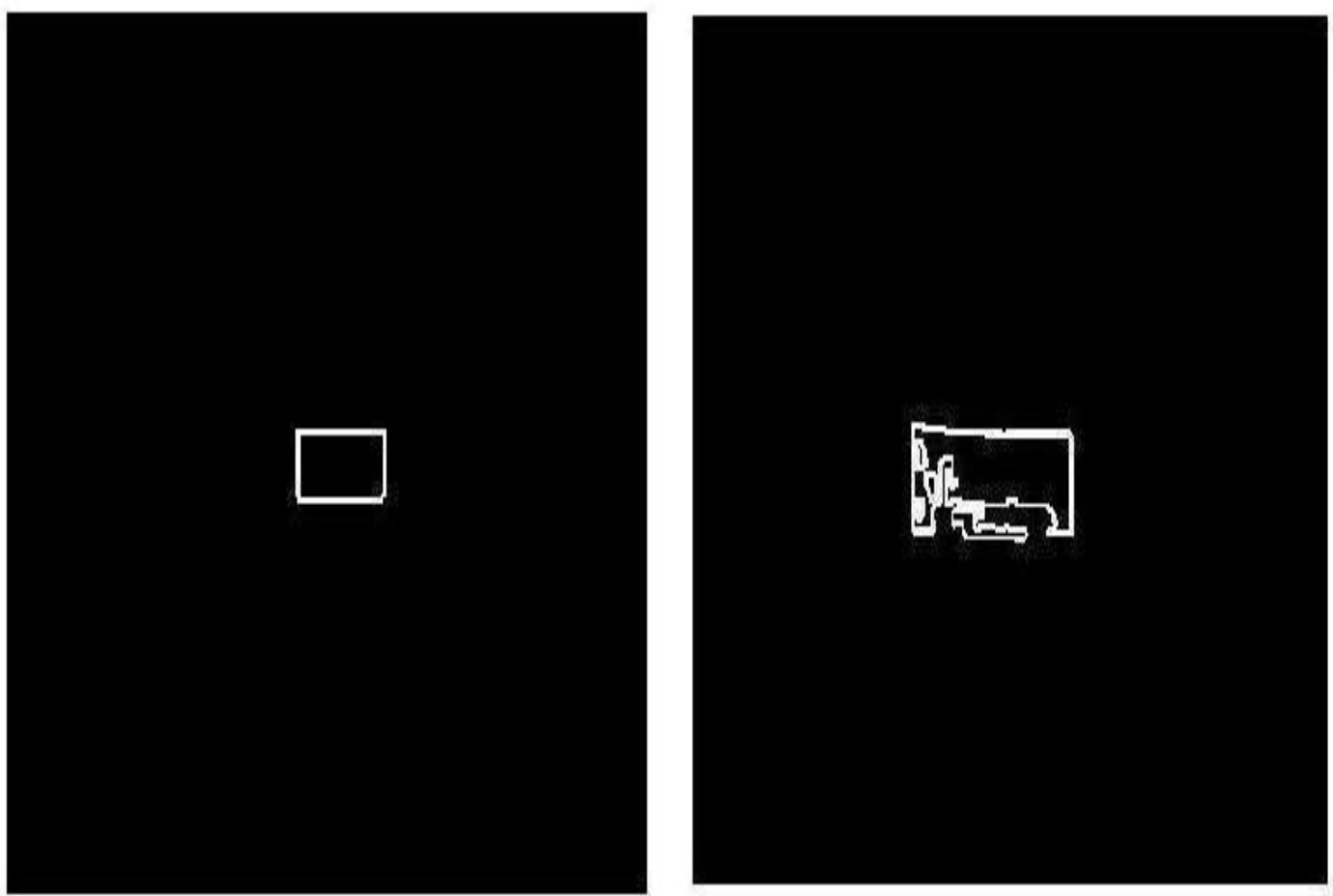

Figure 2.10. Candidate Region

From the above image region of interest is identified as shown in the Figure 2.11. The height of the rectangular portion in the image is 31 pixels and the width of the rectangular portion in the image is 71 pixels.
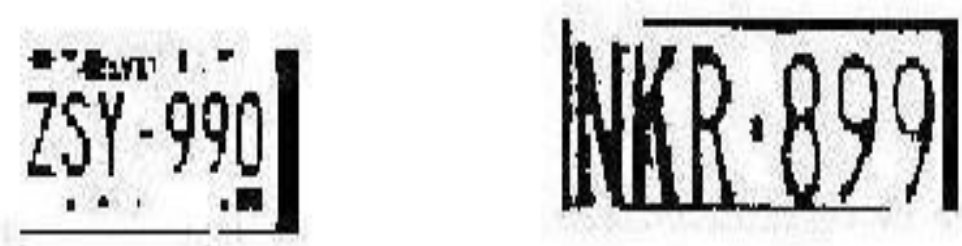

\section{Figure 2.11 Region of Interest}

The final result of RTVT GUI, A car having DS 3C N 5296 uploaded to the GUI of RTVT in Figure 2.12, and the number detected and store in database in Figure 2.13. 


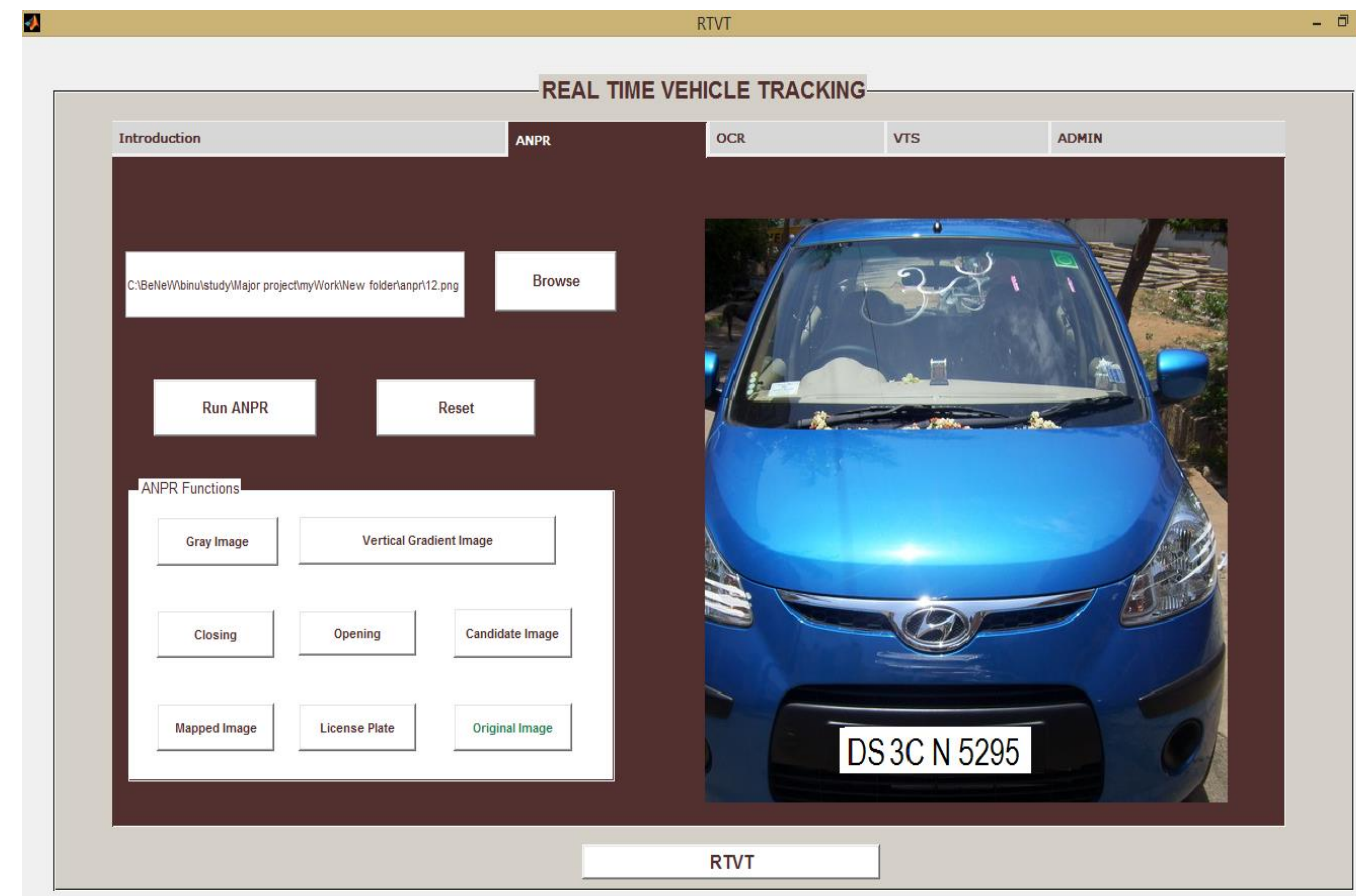

d RTVT

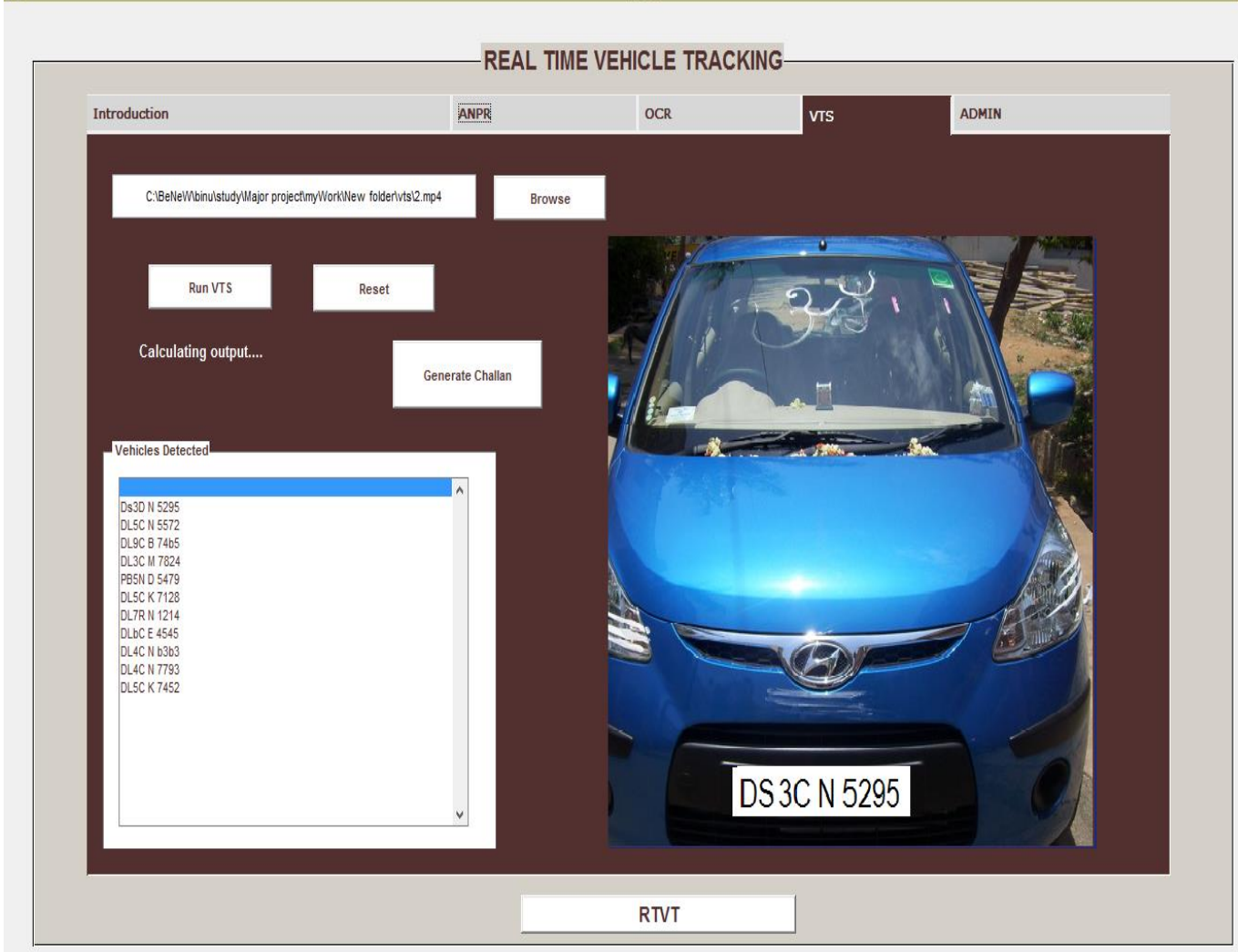

Figure 2.11 and 2.12 Number Store in Database 


\section{Conclusions}

In this RTVT, a statistical threshold is calculated using the HSI model of the image to obtain the binary image of the sample car. Geometrical properties such as aspect ratio, area are used to eliminate the candidate like regions in the license plate. The algorithm provides good results on our database, and it is relatively robust to variations in the luminance conditions and varied kinds of vehicles in different complex environment.

\section{Future Work}

The proposed algorithm can be optimized and it can be further improved by taking into account more parameters and introducing pre-processing and post processing steps which can be applied in order to increase the accuracy of detection. The scope of the project can be extended to consider independent orientation.

\section{References}

[1] Z. Xiehua and Z. Shen, "A robust license plate detection algorithm based on multi-features", The 2nd International Conference on Computer and Automation Engineering (ICCAE), Digital Object Identifier: 10.1109/ICCAE.2010.5451298, vol. 5, (2010), pp. 598 - 602.

[2] O. Serkan and E. Ergun, "Automatic vehicle identification by plate recognition," Proceedings of world academy of science, engineering and technology, vol. 9, (2005), pp. 222- 225.

[3] M. M. I. Chacon and S. A. Zimmerman, "License plate location based on a dynamic PCNN scheme," Proceedings of the international joint conference on neural networks, vol. 2, (2003), pp.1195- 1200.

[4] C. P. Marques, "License vehicle plates localization using maximum correlation", Structural, syntactic, and statistical pattern recognition lecture notes in Computer Science, Springer Berlin / Heidelberg, vol. 3138/2004, (2004), pp. 470-476.

[5] C. Anagnostopoulos, "License plate recognition from still images and video sequence; A Survey", IEEE Transactions on Intelligent Transportation System, vol. 9, no. 3, (2008), pp. 377-391.

[6] Y. Wang, "Study on HSI color model-based fruit quality evaluation", International Congress on Image and Signal Processing (CISP), Digital Object Identifier:10.1109/CISP.2010.5647943, vol. 6, (2010), pp. 2677-2680.

[7] H. Lim, "An efficient method of vehicle license plate detection based on HSI color model and histogram", Next-generation applied intelligence, Digital Object Identifier: 10.1007/978-3-642-02568-6, vol. 5579/2009, (2009), pp. 66-75.

[8] K. Deb, S. Kang and K. Jo, "Statistical characteristics in HSI color model and position histogram based vehicle license plate detection”, Intelligent service robotics, Digital Object Identifier: 10.1007/s11370009-0043-x, vol. 2, (2009), pp. 173-186.

[9] W. Wang, "Reach on Sobel operator for vehicle recognition", International Joint Conference on Artificial Intelligence, IJCAI '09. Digital Object Identifier: 10.1109/JCAI.2009, vol. 54, (2009), pp. 448 $-451$

[10] C. Yan, "Edge detection of images based on improved Sobel operator and genetic algorithms", International Conference on Image Analysis and Signal Processing, IASP 2009. Digital Object Identifier: 10.1109/IASP.2009.5054605, (2009), pp. $31-35$.

[11] M. Donosser, C. Arth and H. Bischof, "Detecting, tracking and recognizing license plates. in: Y.Yagi, S.B.Kang, I.S.Kweon and H.Zha, (eds.) ACCV2--7, Part II. LNCS. Springer, Heidelberg, vol. 4844, (2007), pp. 447-456

[12] Y. Li and M. Wang, "Novel and fast algorithms of license plate locations and extractions," IEEE International Conference on Information and Automation (ICIA), Digital Object Identifier: 10.1109/ICINFA.2010.5512273, (2010), pp. 2462 - 2465.

[13] R. C. Gonzalez, and R. E. Woods, "Digital Image Processing", Third Edition. Prentice Hall, N.J., (2008).

[14] J. Zhang and J. Hu, "Image segmentation based on 2D Otsu method with histogram analysis", International Conference on Computer Science and Software Engineering (CSSE), Digital Object Identifier: 10.1109/CSSE.2008.206, vol. 6, (2008), pp. 105 - 108.

[15] T. Yupeng, "Automatic thresholding of gray-level pictures using two-dimension Otsu method", International Conference on Circuits and Systems (ICCAS), Digital Object Identifier.10.1109/CICCAS.1991.184351, vol.1, (1991), pp. 325 - 327.

[16] B. Hongliang, "A hybrid license plate extraction method based on edge statistics and morphology", Proceedings of $17^{\text {th }}$ International Conference on Pattern Recognition, ICPR 2004, vol. 2, (2004), pp. 831-834

[17] L. Lou,"An efficient method of license plate location", $1^{\text {st }}$ International Conference on Information Science and Engineering(ICISE), vol. 8, (2009), pp. 770-773. 
International Journal of $u-$ and e- Service, Science and Technology

Vol. 10, No. 1 (2017) 